\title{
FIRST EVIDENCE OF A PRECESSING JET EXCAVATING A PROTOSTELLAR ENVELOPE
}

\author{
JASON E. YBARRA ${ }^{1}$ AND MARY BARSONY, ${ }^{1,2}$ \\ Department of Physics and Astronomy, San Francisco State University, 1600 Holloway Drive, San Francisco, CA 94132; \\ jybarra@stars.sfsu.edu, mbarsony@stars.sfsu.edu \\ KARL E. HaISCH, JR. ${ }^{1}$ \\ Utah Valley State College, 800 West University Parkway, Orem, UT 84058; haischka@uvsc.edu \\ THOMAS H. JARRETT ${ }^{1}$ \\ Infrared Processing and Analysis Center, MS 100-22, California Institute of Technology, Pasadena, CA 91125; jarrett@ipac.caltech.edu \\ RAGHVEndRa SAHAI ${ }^{1}$ \\ Jet Propulsion Laboratory, MS 183-900, 4800 Oak Grove Drive, Pasadena, CA 91109; Raghvendra.Sahai@jpl.nasa.gov \\ AND \\ Alycia J. Weinberger \\ Department of Terrestrial Magnetism, Carnegie Institution of Washington, Washington, DC 20015; weinberger@dtm.ciw.edu \\ Received 2006 February 13; accepted 2006 July 5; published 2006 August 8
}

\begin{abstract}
We present new, sensitive, near-infrared images of the Class I protostar, Elias 29, in the Ophiuchus cloud core. To explore the relationship between the infall envelope and the outflow, narrowband $\mathrm{H}_{2} 1-0 S(1), \mathrm{Br} \gamma$, and $K_{\text {cont }}$ filters were used to image the source with the Wide-Field Infrared Camera on the Hale $5 \mathrm{~m}$ telescope and with Persson's Auxiliary Nasmyth Infrared Camera on the Baade $6.5 \mathrm{~m}$ telescope. The source appears as a bipolar, scattered light nebula, with a wide opening angle in all filters, as is typical for late-stage protostars. However, the pure $\mathrm{H}_{2}$ emission-line images point to the presence of a heretofore undetected precessing jet. It is argued that high-velocity, narrow, precessing jets provide the mechanism for creating the observed wide-angled outflow cavity in this source.
\end{abstract}

Subject headings: ISM: jets and outflows — stars: formation — stars: individual (Elias 29)

\section{INTRODUCTION}

Powerful, bipolar supersonic outflows are a hallmark of the earliest protostellar stages. Molecular outflows generally exhibit wide opening angles and velocities of $\sim 10-30 \mathrm{~km} \mathrm{~s}^{-1}$. Optical jets powered by protostars exhibit small opening angles and velocities of $\sim 100-400 \mathrm{~km} \mathrm{~s}^{-1}$ (Mundt et al. 1990; Fridlund et al. 2005). Due to the disparate spatial and velocity distributions of the molecular and optical outflow components, a two-wind structure has often been invoked to explain the instances in which both outflow components are observed to be powered by a single source (Stocke et al. 1988; Davis et al. 2002; Liseau et al. 2005). However, an alternative scenario, in which optical jets and molecular outflows result from a single, collimated flow, is gaining wide acceptance (Hartigan et al. 1994; Sandell et al. 1999; Wolf-Chase et al. 2000; Yun et al. 2001).

An outstanding, unsolved problem with jet-driven outflow models is how jets produce the wide-angle outflow cavities observed in both protostellar envelopes and in molecular outflows. A proposed solution is that of jets changing direction (Masson \& Chernin 1993), for which there is now growing evidence (Bence et al. 1996; Reipurth et al. 1997; Barsony et al. 1998; Arce \& Sargent 2005). Nevertheless, the spatial relationship between the outflow cavity and the currently active wandering jet has not yet been directly observed within the boundaries of a protostellar envelope. We use sensitive, narrowband near-infrared

\footnotetext{
${ }^{1}$ Observations with the Palomar $5 \mathrm{~m}$ telescope were obtained under a collaborative agreement between Palomar Observatory and the Jet Propulsion Laboratory.

${ }^{2}$ Also at Space Science Institute, 4750 Walnut Street, Suite 205, Boulder, CO 80301.
}

imaging to study this relationship: the scattered light emission from the protostellar envelope is imaged with a narrowband continuum filter, whereas the currently active jet regions within the infall envelope are traced in continuum-subtracted, narrowband $\mathrm{H}_{2}$ images, sensitive to shock-excited emission. Based on such observations of the Class I protostar, Elias $29(=$ WL 15 ; $\left.\alpha_{\mathrm{I} 2000.0}=16^{\mathrm{h}} 27^{\mathrm{m}} 09^{\mathrm{s}} 43, \delta_{\mathrm{I} 20000}=-24^{\circ} 37^{\prime} 18^{\prime \prime} .7\right)$ in the nearby $(d=125 \mathrm{pc}) \rho$ Ophiuchi molecular cloud, we report the first detection of a precessing jet carving out a protostellar envelope's cavity.

\section{OBSERVATIONS AND DATA REDUCTION}

Near-infrared, narrowband imaging of Elias 29 was undertaken at two facilities, Palomar and Las Campanas observatories. The Wide Field Infrared Camera (WIRC) on the Hale $5 \mathrm{~m}$ telescope at Palomar Observatory, with its 8.7 $\times$ 8.7 field of view, was used to image the field including Elias 29 (Wilson et al. 2003). WIRC employs a $2048 \times 2048$ pixel Hawaii-II detector with a plate scale of 0 ."2487 pixel $^{-1}$. The $K$-band seeing was $\sim 1$ ".0 on the night of the observations, 2004 July 11 . Two narrowband filters were used for imaging: an $\mathrm{H}_{2} 1-0 S(1)$ filter centered at $2.120 \mu \mathrm{m}$ with a $1.5 \%$ bandpass and a line-free continuum filter $\left(K_{\text {cont }}\right)$ centered at $2.270 \mu \mathrm{m}$ with a $1.7 \%$ bandpass. The telescope was dithered in a nine-point pattern, with $6^{\prime \prime}$ offsets between dither positions. The integration time at each dither position was $60 \mathrm{~s}$ ( $30 \mathrm{~s} \times 2$ co-adds). The dither pattern was repeated, after a $5^{\prime \prime}$ offset of the telescope, until a total integration time of 27 minutes was reached in each filter. Elias 29 was reobserved with Persson's Auxiliary Nasmyth Infrared Camera (PANIC) on the Baade $6.5 \mathrm{~m}$ telescope at Las Campanas Observatory (LCO) on the night of 2005 June 17 UT. The $K$ band seeing was 0"5. PANIC employs a $1024 \times 1024$ Hawaii- 


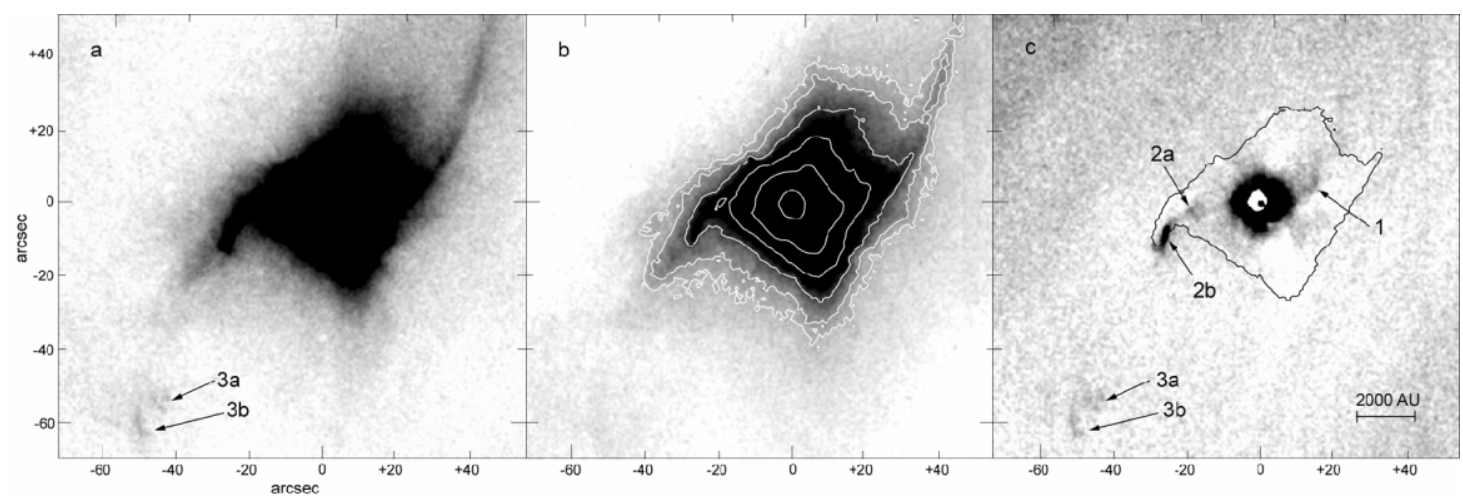

FIG. 1.-Narrowband images of Elias 29. North is up and east is left in all panels. Offsets from $\alpha_{\mathrm{J} 2000.0}=16^{\mathrm{h}} 27^{\mathrm{m}} 09^{\mathrm{s}} .43$ and $\delta_{\mathrm{J} 2000.0}=-24^{\circ} 37^{\prime} 18^{\prime \prime} 7$ are indicated, in arcseconds. (a) Elias 29 observed through the narrowband $\mathrm{H}_{2}$ filter with PANIC. (b) Elias 29 imaged with the Br $\gamma$ filter with PANIC; contour levels are 3, 5, $10,20,50$, and $500 \sigma$. Note the absence of features $3 \mathrm{a}$ and $3 \mathrm{~b}$ in the $\mathrm{Br} \gamma$ image and their presence in the $\mathrm{H}_{2}$ filter image. $(c)$ Pure $\mathrm{H}_{2}$ emission-line image of Elias 29, obtained by subtracting the scaled and aligned $\mathrm{Br} \gamma$ filter image from the PANIC $\mathrm{H}_{2}$ filter image with the $\mathrm{Br} \gamma 10 \sigma$ contour superposed. The pure $\mathrm{H}_{2}$ emission-line features discussed in the text and listed in Table 1 are labeled.

I detector with a plate scale of 0.125 pixel $^{-1}$ (Martini et al. 2004). The two narrowband filters used for imaging at $\mathrm{LCO}$ were the $\mathrm{H}_{2} 1-0 \mathrm{~S}(1)$ filter, centered at $2.125 \mu \mathrm{m}$ with a $1.1 \%$ bandpass, and the $\mathrm{Br} \gamma$ filter, centered at a wavelength of $2.165 \mu \mathrm{m}$ with a $1.0 \%$ bandpass. The $\mathrm{Br} \gamma$ filter served as a surrogate narrowband continuum filter for the PANIC data set, in the absence of a linefree, narrowband continuum filter adjacent to the $\mathrm{H}_{2}$ filter at LCO. The observing pattern for Elias 29 at LCO consisted of interleaved on- and off-source integrations of $30 \mathrm{~s}$ duration each through each filter. On-source integrations were dithered in a checkerboard pattern, with $12^{\prime \prime}$ offsets. Five-point dithered sky observations were taken in a clear patch $3^{\prime}$ east of Elias 29. The final on-source integration times with PANIC were 70 minutes through the $\mathrm{H}_{2}$ filter and 67.5 minutes through the $\mathrm{Br} \gamma$ filter.

All data were reduced using IRAF. ${ }^{3}$ Object frames were skysubtracted, flat-fielded, and corrected for bad pixels for each night's observations through each narrowband filter. Individual processed images for each filter/instrument combination were then aligned using the available point sources in common with the IRAF task IMCENTROID, before combining to produce final images.

Although the $\mathrm{H}_{2}$ filters are so-called because they transmit the $2.12 \mu \mathrm{m}$ emission line, they both also transmit some continuum emission if present in the source. Therefore, to trace the morphology of any outflow/jet component within the protostellar envelope of Elias 29, the bright continuum emission from the scattering envelope must be subtracted from the $\mathrm{H}_{2}$ filter images. This requires proper scaling and alignment of the narrowband continuum images before subtracting them from the respective $\mathrm{H}_{2}$ filter images. These tasks were achieved separately for the WIRC and PANIC data. For the PANIC images, shown in Figure 1, the $\mathrm{Br} \gamma$ filter image was scaled by a factor of 0.82 , which is simply the ratio of the transmission of the $\mathrm{H}_{2}$ filter to that of the $\mathrm{Br} \gamma$ filter, from the filter transmission curves. Proper alignment of the $\mathrm{H}_{2}$ and scaled continuum images before subtraction, crucial for obtaining accurate pure $\mathrm{H}_{2}$ line-emission images, was achieved by using the IRAF task IMCENTROID for common point sources in each filter.

For astrometry, a plate solution was determined for the WIRC data by using a least-squares fit for seven pointlike

\footnotetext{
${ }^{3}$ IRAF is distributed by the National Optical Astronomy Observatory, which is operated by the Association of Universities for Research in Astronomy (AURA), Inc., under cooperative agreement with the National Science Foundation (NSF).
}

objects in common with the Two Micron All Sky Survey (2MASS). ${ }^{4}$ The 2 MASS reference objects had uncertainty ellipses of $\sim 0^{\prime \prime} 09$. The plate solution had a $00^{\prime \prime} 2 \mathrm{rms}$ residual, our estimated astrometric uncertainty.

\section{RESULTS AND DISCUSSION}

The reduced WIRC and PANIC images of Elias 29 appear essentially identical. Only the PANIC images are presented here, since these were obtained in better seeing and have higher signal-to-noise ratio $(\mathrm{S} / \mathrm{N})$. Figure 1 shows our results, all presented at a linear gray-scale stretch to emphasize the presence of the pure emission features, $3 \mathrm{a}$ and $3 \mathrm{~b}$ in Figure $1 a$, and their absence from Figure $1 b$. Figure $1 a$ shows the appearance of Elias 29 through the narrowband $\mathrm{H}_{2}$ filter. Figure $1 b$ shows Elias 29 through the surrogate narrowband continuum $(\mathrm{Br} \gamma)$ filter. Since the NIR continuum emission overwhelms the strength of the pure $\mathrm{H}_{2}$ line emission for this object, Elias 29 looks similar in both filters. Figure $1 c$ shows the continuumsubtracted, pure $\mathrm{H}_{2}$ emission-line image of Elias 29. Saturation effects from the extreme brightness of Elias 29 prevent correct image subtraction within the central $\sim 5^{\prime \prime} .5$ radius region, resulting in the black and white circular artifacts at the center of Figure 1c. Outside this area, five regions of pure $\mathrm{H}_{2}$ line emission are discovered, having been missed by previous investigators (Gómez et al. 2003; Khanzadyan et al. 2004). The appearance of Elias 29 in Figures $1 a$ and $1 b$ is consistent with scattered light models of Class I protostars consisting of an infall envelope containing a bipolar cavity (Whitney et al. 2003). The boundary of the wide-angled cavity walls within the protostellar infall envelope is indicated by the $10 \sigma$ contour levels overplotted on Figures $1 b$ and $1 c$. The lack of a narrow "waist," or distinctive hourglass shape, at this angular resolution is consistent with a disk of small spatial extent (B. Whitney 2005, private communication). Elias 29 appears strikingly different in the continuum-subtracted, pure $\mathrm{H}_{2}$ emission-line image than in the narrowband filter images. The pure $\mathrm{H}_{2}$ emission objects, labeled 1, 2a, 2b, 3a, and 3b, appear at the same location and have similar morphologies in both the PANIC and the WIRC (not shown here) continuum-subtracted images. Taken together, the $\mathrm{H}_{2}$ emission knots form a sinuous structure with S-shaped point symmetry about the center.

Table 1 lists the observed characteristics of each $\mathrm{H}_{2}$ feature.

${ }^{4}$ See http://www.phys.vt.edu/ jhs/SIPbeta/astrometrycalc.html. 
TABLE 1

$\mathrm{H}_{2}$ EMISSION FEATURES

\begin{tabular}{ccccr}
\hline \hline $\begin{array}{c}\mathrm{H}_{2} \text { Feature } \\
\text { Designation } \\
(1)\end{array}$ & $\begin{array}{c}\alpha \\
(\mathrm{J} 2000.0) \\
(2)\end{array}$ & $\begin{array}{c}\delta \\
(\mathrm{J} 2000.0) \\
(3)\end{array}$ & $\begin{array}{c}\text { Aperture } \\
(\operatorname{arcsec}) \\
(4)\end{array}$ & $\begin{array}{r}\text { S/N } \\
(5)\end{array}$ \\
\hline $1 \ldots \ldots \ldots \ldots$ & 162708.79 & -243716.5 & $0.6 \times 0.5$ & 3.5 \\
$2 \mathrm{a} \ldots \ldots \ldots \ldots$ & 162710.04 & -243720.8 & $0.6 \times 0.5$ & 3.9 \\
$2 \mathrm{~b} \ldots \ldots \ldots$. & 162710.70 & -243724.3 & $1.3 \times 0.5$ & 14.2 \\
$3 \mathrm{a} \ldots \ldots \ldots$. & 162711.64 & -243756.1 & $0.6 \times 0.5$ & 2.6 \\
$3 \mathrm{~b} \ldots \ldots \ldots$. & 162711.88 & -243800.0 & $0.9 \times 0.4$ & 2.8 \\
\hline
\end{tabular}

Note.-Units of right ascension are hours, minutes, and seconds, and units of declination are degrees, arcminutes, and arcseconds.

The first column lists the feature designations from Figure $1 c$. The second and third columns list the coordinates of each $\mathrm{H}_{2}$ emission region, corresponding to the WIRC pixel with the highest counts in each object. The coordinates are derived from the WIRC data, since there were not enough sources in the smaller PANIC field to provide a plate solution for absolute astrometry. The final, continuum-subtracted PANIC image was used to determine the $\mathrm{S} / \mathrm{N}$ of each emission feature, listed in the column (5) of Table 1 , since the seeing and $\mathrm{S} / \mathrm{N}$ were better in the PANIC than in the WIRC data set. A rectangular aperture (of dimensions given in col. [4] of Table 1) was used to determine the average counts in each feature. Several background regions were used to calculate the average background noise level and its standard deviation. The background level was subtracted from the average counts in the rectangular aperture containing each $\mathrm{H}_{2}$ emission region, and the result was divided by the standard deviation of the background to give the $\mathrm{S} / \mathrm{N}$. Only features $3 \mathrm{a}$ and $3 \mathrm{~b}$ have $\sigma<3$. Nevertheless, these objects are real, since they appear at identical locations and have similar morphologies in both data sets (WIRC and PANIC).

$\mathrm{H}_{2}$ emission associated with molecular outflows generally arises from shock interactions of the unseen, fast jet/wind component with the ambient medium. Elias 29 is known to drive a CO outflow (Bontemps et al. 1996; Sekimoto et al. 1997) with velocities as high as $\sim 80 \mathrm{~km} \mathrm{~s}^{-1}$ (Boogert et al. 2002), although its morphology is unknown at the scales of the images presented here. In some Class I protostellar envelopes, both spatially diffuse $\mathrm{H}_{2}$ emission and compact $\mathrm{H}_{2}$ emission knots have been identified in the same source (Yamashita \& Tamura 1992; Davis et al. 2002). In such cases, the diffuse component is found to trace the infrared continuum reflection nebula, whereas knotty, compact emission traces the fast jets observed at optical or centimetercontinuum wavelengths. By analogy, the knotty $\mathrm{H}_{2}$ emission features identified in Figure $1 c$ are likely to be associated with the jets driven by Elias 29. Since the cooling time of shocked $2.12 \mu \mathrm{m} \mathrm{H}_{2}$ emission is of the order of about a few years (Shull \& Beckwith 1982; Allen \& Burton 1993), the newly identified features in Figure $1 c$ are tracing recent shock activity. The three $\mathrm{H}_{2}$ emission features in Figure $1 c(1,2 \mathrm{a}$, and $2 \mathrm{~b})$ describe an S-shaped, point symmetry about the center, consistent with a precessing jet. Features $3 \mathrm{a}$ and $3 \mathrm{~b}$ are extended and diffuse, close to the inferred cavity wall within the southern hemisphere of the protostellar envelope of Elias 29. More quantitative results on the precessing jet, such as its precession period and initial opening angle await Hubble Space Telescope NICMOS polarimetry. Ground-based, AO-assisted integral field spectroscopy of the $\mathrm{H}_{2}$ line would establish the velocity field.

Hydrodynamic simulations of molecular outflows driven by pulsed, precessing protostellar jets have become available only recently (Lim 2001; Rosen \& Smith 2004; Smith \& Rosen 2005). One stated aim of these studies is to model the outflows to infer properties of the (unseen) driving jets, such as jet power and precession. Currently, only a limited set of models have been calculated with accompanying simulated $\mathrm{H}_{2}$ images. Assumed jet radii are $1.7 \times 10^{15} \mathrm{~cm}$, corresponding to $\sim 1$ ". 0 at the distance to Elias 29 (thus well beyond the disk region where jets may be generated). The pulsation is input as a $30 \%$ velocity modulation with a $60 \mathrm{yr}$ period into the models. The time span over which the models are calculated is short $(t<500 \mathrm{yr})$, but it is much longer than the dynamical time of a few decades for a jet traveling a conservative $\sim 100 \mathrm{~km} \mathrm{~s}^{-1}$ to reach the $\mathrm{H}_{2}$ features 1 and $2 \mathrm{a}$ in Elias 29. This, compared with the very fast cooling time of $\mathrm{H}_{2}$, validates comparison of the Elias 29 pure emission-line $\mathrm{H}_{2}$ image (Fig. 1c) with simulated $\mathrm{H}_{2}$ images.

Models of molecular jets with precession angles of $5^{\circ}, 10^{\circ}$, and $20^{\circ}$ have been calculated, although the precession angle of the Elias 29 jet may well be even wider, based on the projected half-angles formed by features 1 and $2 \mathrm{a}$ with the central symmetry axis of the observed reflection nebulosity. Simulated $\mathrm{H}_{2}$ images from two general classes of precessing jet models have been published: jets with assumed precession periods of $50 \mathrm{yr}$, termed "fast" precessing, and jets with precession periods of $400 \mathrm{yr}$, termed "slow" precessing (Rosen \& Smith 2004; Smith $\&$ Rosen 2005). The terms fast and slow here are relative to the flow evolution time of 500 yr. Comparison of Figure 1c with synthetic $\mathrm{H}_{2} v=1-0 \mathrm{~S}(1)$ model images from such simulations leads to the conclusion that this jet exhibits slow, rather than fast, precession; that is to say, qualitatively, Figure $1 c$ resembles the $\mathrm{H}_{2} 1 \rightarrow 0$ morphologies presented in Figures 6 and 7, rather than those of Figure 5, of Smith \& Rosen (2005). For slow precession, the simulations show ordered chains of bow shocks and meandering streamers of $\mathrm{H}_{2}$, which contrast with the chaotic $\mathrm{H}_{2}$ structures produced by jets in rapid precession. The production of specific simulated $\mathrm{H}_{2}$ images for direct comparison with Figure $1 c$ is beyond the scope of this Letter but may be attempted in the future.

Images of both scattered light cavities and $\mathrm{H}_{2}$ jet emission within them exist for only a handful of Class I objects (Davis et al. 2002). For all of these, the jets lie well within the outflow cavity walls, close to, if not precisely along, the symmetry axis of each outflow cavity. By contrast, the jet of Elias 29, as traced by $\mathrm{H}_{2}$, is found close to, and, in one case (feature 2b) along, the cavity walls (Fig. 1c).

Examples of other S-shaped, precessing outflows are known (Gueth et al. 1996; Schwartz \& Greene 1999; Hodapp et al. 2005; Sahai et al. 2005). However, no precessing protostellar jets have been imaged at their base within their scattered light cavities, either because they are too embedded to be detected in the NIR (L1157, HH 211-mm) or because of lack of narrowband continuum data excluding the $\mathrm{H}_{2}$ emission line for proper image subtraction (IRAS $03256+3055$ ). Binarity has inevitably been invoked to account for the presence of precessing jets (Terquem et al. 1999). However, Elias 29 is known to be single (Simon et al. 1995; Ghez et al. 1993). At scales between $\sim 1-60$ AU, lunar occultation observations show that $90 \%$ of the $K$-band flux from Elias 29 originates in a component $\sim 7$ mas diameter $(\sim 0.8 \mathrm{AU})$, with the remaining $10 \%$ of the $K$-band flux originating in a much larger, 415 mas diameter, diffuse component (Simon et al. 1987). It is then inferred that within $\approx 0.8 \mathrm{AU}$, Elias 29 is a single object. Alternative scenarios leading to jet precession that may be operative in Elias 29 might be disk oscillations associated with a propeller-driven outflow, recently discovered in MHD simulations (Romanova et al. 2005), or warping of the accretion disk caused by the magnetically driven jets (Lai 2003). Future high-resolution in- 
tegral field spectroscopic observations of both the jet and the disk components could test the latter model, which predicts the disk and jets to precess in opposite senses.

We thank our very professional night assistants, Jean Mueller at Palomar Observatory and Hernan Nuñez, at Las
Campanas Observatory. Portions of this research were funded by NSF AST 02-06146 to M. B. and by the NASA LTSA Program (NAG5-8933). NASA/LTSA Award 399-3061-00-00 to R. S. is gratefully acknowledged. A. J. W. acknowledges support from NASA Space Science grant NAG5-3175.

\section{REFERENCES}

Allen, D. A., \& Burton, M. G. 1993, Nature, 363, 54

Arce, H. G., \& Sargent, A. I. 2005, ApJ, 624, 232

Barsony, M., Ward-Thompson, D., André, Ph., \& O’Linger, J. 1998, ApJ, 509, 733

Bence, S., Richer, J. S., \& Padman, R. 1996, MNRAS, 279, 866

Bontemps, S., André, P., Terebey, S., \& Cabrit, S. 1996, A\&A, 311, 858

Boogert, A. C. A., Hogerheijde, M. R., Ceccarelli, C., Tielens, A. G. G. M., van Dishoeck, E. F., Blake, G. A., Latter, W. B., \& Motte, F. 2002, ApJ, 570, 708

Davis, C. J., Stern, L., Ray, T. P., \& Chrysostomou, A. 2002, A\&A, 382, 1021 Fridlund, C. V. M., Liseau, R., Djupvik, A. A., Huldtgren, M., White, G. J., Favata, F., \& Giardino, G. 2005, A\&A, 436, 983

Ghez, A., Neugebauer, G., \& Matthews, K. 1993, AJ, 106, 2005

Gómez, M., Stark, D. P., Whitney, B. A., \& Churchwell, E. 2003, AJ, 126, 863

Gueth, F., Guilloteau, S., \& Bachiller, R. 1996, A\&A, 307, 891

Hartigan, P., Morse, J., \& Raymond, J. 1994, ApJ, 436, 125

Hodapp, K. W., Bally, J., Eislöffel, J., \& Davis, C. J. 2005, AJ, 129, 1580

Khanzadyan, T., Gredel, R., Smith, M. D., \& Stanke, T. 2004, A\&A, 426, 171

Lai, D. 2003, ApJ, 591, L119

Lim, A. J. 2001, MNRAS, 327, 507

Liseau, R., Fridlund, C. V. M., \& Larsson, B. 2005, ApJ, 619, 959

Martini, P., Persson, S. E., Murphy, D. C., Birk, C., Shectman, S. A., Gunnels, S. M., \& Koch, E. 2004, Proc. SPIE, 5492, 1653

Masson, C. R., \& Chernin, L. 1993, ApJ, 414, 230

Mundt, R., Bührke, T., Solf, J., Ray, T. P., \& Raga, A. C. 1990, A\&A, 232, 37
Reipurth, B., Bally, J., \& Devine, D. 1997, AJ, 114, 2708

Romanova, M. M., Ustyugova, G. V., Koldoba, A. V., \& Lovelace, R. V. E. 2005, ApJ, 635, L165

Rosen, A., \& Smith, M. D. 2004, MNRAS, 347, 1097

Sahai, R., Le Mignant, D., Sánchez Contreras, C., Campbell, R. D., \& Chaffee, F. H. 2005, ApJ, 622, L53

Sandell, G., et al. 1999, ApJ, 519, 236

Schwartz, R. D., \& Greene, T. P. 1999, AJ, 117, 456

Sekimoto, Y., Tatematsu, K., Umemoto, T., Koyama, K., Tsuboi, Y., Hirano, N., \& Yamamoto, S. 1997, ApJ, 489, L63

Shull, J. M., \& Beckwith, S. 1982, ARA\&A, 20, 163

Simon, M., Howell, R. R., Longmore, A. J., Wilking, B. A., Peterson, D. M., \& Chen, W. P. 1987, ApJ, 320, 344

Simon, M., et al. 1995, ApJ, 443, 625

Smith, M. D., \& Rosen, A. 2005, MNRAS, 357, 579

Stocke, J., Hartigan, P. M., Strom, S. E., Strom, K. M., Anderson, E. R., Hartmann, L., \& Kenyon, S. J. 1988, ApJS, 68, 229

Terquem, C., Eislöffel, J., Papaloizou, J., \& Nelson, R. P. 1999, ApJ, 512, L131

Whitney, B. A., Wood, K., Bjorkman, J. E., \& Cohen, M. 2003, ApJ, 598, 1079

Wilson, J. E., et al. 2003, Proc. SPIE, 4841, 451

Wolf-Chase, G. A., Barsony, M., \& O’Linger, J. 2000, AJ, 120, 1467

Yamashita, T., \& Tamura, M. 1992, ApJ, 387, L93

Yun, J. C., Santos, C. A., Clemens, D. P., Afonso, J. M., McCaughrean, M. J., Preibisch, T., Stanke, T., \& Zinnecker, H. 2001, A\&A, 372, L33 